\title{
CONVERSES TO MEASURABILITY THEOREMS FOR YEH-WIENER SPACE
}

\author{
DAVID SKOUG
}

\begin{abstract}
In this paper we establish some relationships between YehWiener measurability and Wiener measurability of certain sets and functionals. In addition we show that an "interval" in Yeh-Wiener space is YehWiener measurable if and only if its "restriction set" in Euclidean space is Lebesgue measurable.
\end{abstract}

0. Introduction. Let $C_{1}[a, b]$ denote the Wiener space of one variable, i.e. $C_{1}[a, b]=\{x(\cdot) \mid x(a)=0$ and $x(s)$ is continuous on $[a, b]\}$. Let $R=\{(s, t) \mid$ $a \leqslant s \leqslant b, a<t<\beta\}$ and let $C_{2}[R]$, called Yeh-Wiener space, denote the Wiener space of functions of two variables over $R$, i.e. $C_{2}[R]=\{x(\cdot, \cdot) \mid$ $x(a, t)=x(s, \alpha)=0$ and $x(s, t)$ is continuous on $R$.

In [2] Cameron and Storvick evaluate certain Yeh-Wiener integrals in terms of Wiener integrals. In particular they obtain the following theorem (this theorem also plays a key role in [3]);

THEOREM A. Let $\alpha<\gamma \leqslant \beta$ and let $f$ be a real or complex valued functional defined on $C_{1}[a, b]$ such that $f\left([(\gamma-\alpha) / 2]^{1 / 2} y\right)$ is a Wiener measurable functional of $y$ on $C_{1}[a, b]$. Then $f(x(\cdot, \gamma))$ is a Yeh-Wiener measurable functional of $x(\cdot, \cdot)$ on $C_{2}[R]$ and

$$
\int_{C_{2}[R]} f(x(\cdot, \gamma)) d x=\int_{C_{1}[a, b]} f\left([(\gamma-\alpha) / 2]^{1 / 2} y\right) d y
$$

where the existence of either integral implies the existence of the other and their equality.

Since one is trying to evaluate Yeh-Wiener integrals it would seem very natural and desirable to assume that the functional $f(x(\cdot, \gamma))$ is a Yeh-Wiener measurable functional of $x(\cdot, \cdot)$ on $C_{2}[R]$ and conclude that $f\left([(\gamma-\alpha) / 2]^{1 / 2} y\right)$ is a Wiener measurable functional of $y$ on $C_{1}[a, b]$. This would also allow the proof of equation (1) to proceed in the same order as the motivation; where as in [2] the proof had to proceed in the opposite order from the motivation because of the measurability argument. We obtain this result (i.e. the converse of Theorem $\mathrm{A}$ ) in $\$ 2$ below. In particular we show that if $A$ is any subset of $C_{1}[a, b]$ and if $B_{A} \equiv\left\{x(\cdot, \cdot) \in C_{2}[R] \mid x(\cdot, \gamma) \in A\right\}$ then $B_{A}$ is a Yeh-Wiener

Received by the editors August 28, 1975.

AMS (MOS) subject classifications (1970). Primary 28A40.

Key words and phrases. Wiener integral, Yeh-Wiener integral. 
measurable subset of $C_{2}[R]$ if and only if $[2 /(\gamma-\alpha)]^{1 / 2} A$ is a Wiener measurable subset of $C_{1}[a, b]$.

Let $a=s_{0}<s_{1}<\cdots<s_{m}=b$ and $\alpha=t_{0}<t_{1}<\cdots<t_{n}=\beta$ be subdivisions of $[a, b]$ and $[\alpha, \beta]$ respectively. Let $E$ be any subset of Euclidean space $R^{m n}$ and let $Q=\left\{x \in C_{2}[R] \mid\left\langle x\left(s_{1}, t_{1}\right), \ldots, x\left(s_{m}, t_{n}\right)\right\rangle \in E\right\}$. By the definition of Yeh-Wiener measure, if $E$ is Lebesgue measurable then $Q$ is YehWiener measurable. In $\$ 4$ we establish the converse; i.e. if $Q$ is Yeh-Wiener measurable then $E$ is Lebesgue measurable. In an unpublished result Fulton Koehler established this fact for $C_{1}[a, b]$.

The techniques used in this paper are quite different from the technique used in [2] and [4]. The key to obtaining these "converse theorems" is Lemma 3 in which we show that the outer Yeh-Wiener measure of the set $B_{A}$ is equal to the outer Wiener measure of the set $[2 /(\gamma-\alpha)]^{1 / 2} A$.

1. Preliminaries. In this section, for the convenience of the reader, we will present a brief discussion of Wiener measure on $C_{1}[a, b]$ and Yeh-Wiener measure on $C_{2}[R]$.

Let $a=s_{0}<s_{1}<\cdots<s_{m}=b$ and let $E$ be a Lebesgue measurable set in Euclidean $m$-space $R^{m}$. Then

$$
I=\left\{y(\cdot) \in C_{1}[a, b] \mid\left\langle y\left(s_{1}\right), \ldots, y\left(s_{m}\right)\right\rangle \in E\right\}
$$

is called an interval in $C_{1}[a, b]$. The Wiener measure of the interval $I$ is defined to be

$$
m_{1}(I) \equiv \int_{E} W(\vec{u} ; \vec{s}) d \vec{u}
$$

where

$$
\begin{aligned}
W(\vec{u} ; \vec{s}) & =W\left(u_{1}, \ldots, u_{m} ; s_{1}, \ldots, s_{m}\right) \\
& =\prod_{j=1}^{m}\left[2 \pi\left(s_{j}-s_{j-1}\right)\right]^{-1 / 2} \exp \left\{\frac{-\left(u_{j}-u_{j-1}\right)^{2}}{2\left(s_{j}-s_{j-1}\right)}\right\}
\end{aligned}
$$

and $u_{0} \equiv 0$. This measure is countably additive on the set of all such intervals in $C_{1}[a, b]$. The outer Wiener measure $m_{1}^{*}$ of any subset of $C_{1}[a, b]$ is now defined in the usual way and the term "Wiener measurable set" will denote any set which is measurable with respect to outer Wiener measure. We let $\mathfrak{\pi}_{1}$ denote the class of all Wiener measurable sets and $m_{1}^{*}$ restricted to $\mathfrak{R}_{1}$ will be denoted by $m_{1}$. Integration of a functional $F$ with respect to Wiener measure $m_{1}$ will be denoted by $\int_{C_{1}[a, b]} F(y) d y$.

Let $a=s_{0}<s_{1}<\cdots<s_{m}=b, \alpha=t_{0}<t_{1}<\cdots<t_{n}=\beta$, and let $E$ be a Lebesgue measurable set in Euclidean space $R^{m n}$. Then

$$
J=\left\{x(\cdot, \cdot) \in C_{2}[R] \mid\left\langle x\left(s_{1}, t_{1}\right), \ldots, x\left(s_{m}, t_{n}\right)\right\rangle \in E\right\}
$$

is called an interval in $C_{2}[R]$. The Yeh-Wiener measure of the interval $J$ is defined to be

$$
m_{2}(J) \equiv \int_{E} W(\vec{u} ; \vec{s} ; \vec{t}) d \vec{u}
$$

where 


$$
\begin{aligned}
W(\vec{u} ; \vec{s} ; \vec{t})= & W\left(u_{1,1}, \ldots, u_{m, n} ; s_{1}, \ldots, s_{m} ; t_{1}, \ldots, t_{n}\right) \\
= & \prod_{j=1}^{m} \prod_{k=1}^{n}\left[\pi\left(s_{j}-s_{j-1}\right)\left(t_{k}-t_{k-1}\right)\right]^{-1 / 2} \\
& \cdot \exp \left\{\frac{-\left(u_{j, k}-u_{j-1, k}-u_{j, k-1}+u_{j-1, k-1}\right)^{2}}{\left(s_{j}-s_{j-1}\right)\left(t_{k}-t_{k-1}\right)}\right\}
\end{aligned}
$$

and $u_{0, k}=u_{j, 0}=u_{0,0}=0$ for all $j$ and $k$. This measure is countably additive on the set of all such intervals in $C_{2}[R]$. The outer Yeh-Wiener measure $m_{2}^{*}$ of any subset of $C_{2}[R]$ is now defined in the usual way and the term "Yeh-Wiener measurable set" will denote any set which is measurable with respect to outer Yeh-Wiener measure. We let $\mathfrak{\Re}_{2}$ denote the class of all Yeh-Wiener measurable sets and $m_{2}^{*}$ restricted to $\Re_{2}$ will be denoted by $m_{2}$. Integration of a functional $F$ with respect to Yeh-Wiener measure $m_{2}$ will be denoted by $\int_{C_{2}[R]} F(x) d x$.

2. Some relationships between Yeh-Wiener and Wiener measurability. Our first theorem in this section (whose rather lengthy proof is given in \$3) establishes a relationship between Yeh-Wiener measurability and Wiener measurability of certain related sets.

THEOREM 1. Let $\alpha<\gamma \leqslant \beta$, let $A$ be any subset of $C_{1}[a, b]$ and let $B_{A} \equiv\left\{x \in C_{2}[R] \mid x(\cdot, \gamma) \in A\right\}$. Then $B_{A} \in \mathscr{N}_{2}$ if and only if $[2 /(\gamma-\alpha)]^{1 / 2} A$ $\in \Re_{1}$. Furthermore the equation

$$
\begin{aligned}
m_{2}\left(B_{A}\right) & =\int_{C_{2}[R]} \chi_{A}(x(\cdot, \gamma)) d x \\
& =\int_{C_{1}[a, b]} \chi_{[2 /(\gamma-\alpha)]^{1 / 2} A}(y) d y=m_{1}\left([2 /(\gamma-\alpha)]^{1 / 2} A\right)
\end{aligned}
$$

holds if either $B_{A} \in \mathfrak{N}_{2}$ or $[2 /(\gamma-\alpha)]^{1 / 2} A \in \mathfrak{R}_{1}$.

Theorem 2. Let $\gamma, A$ and $B_{A}$ be as in Theorem 1.

(a) If $\gamma-\alpha=2$ then $B_{A} \in \Re_{2} \Leftrightarrow A \in \Re_{1}$.

(b) If $\gamma-\alpha \neq 2$ then $A \in \Re_{1} \neq B_{A} \in \Re_{2}$.

(c) If $\gamma-\alpha \neq 2$ then $B_{A} \in \Re_{2} \neq A \in \Re_{1}$.

Proof. Statement (a) is a special case of Theorem 1. To establish (b) let $H$ be a subset of $C_{1}[a, b]$ with the property that $m_{1}(H)=1$ and $m_{1}(\lambda H)=0$ for all real $\lambda \neq \pm 1$. (For the existence of such a set see [1].) Let $G$ be a nonmeasurable subset of $C_{1}[a, b]$. Then $H \cap G \notin \Re_{1}$. Let

$$
A=[(\gamma-\alpha) / 2]^{1 / 2}(H \cap G) .
$$

Then $A$ is a iull set in $C_{1}[a, b]$ and so $A \in \Re_{1}$. But then $[2 /(\gamma-\alpha)]^{1 / 2} A$ $=H \cap G \notin \Re_{1}$ and so by Theorem $1, B_{A} \notin \Re_{2}$. Hence $A \in \pi_{1} \neq B_{A}$ $\in \mathbb{R}_{2}$.

To establish (c) let $A=H \cap G$ where $H$ and $G$ are as above. Then $A \notin \mathfrak{N}_{1}$. But $[2 /(\gamma-\alpha)]^{1 / 2} A=[2 /(\gamma-\alpha)]^{1 / 2}(H \cap G)$ is a null set in $C_{1}[a, b]$, hence in $\mathfrak{R}_{1}$ and so by Theorem $1, B_{A} \in \mathfrak{N}_{2}$. Hence $B_{A} \in \mathfrak{N}_{2} \nRightarrow A$ $\in \pi_{1}$. 
THEOREM 3. Let $\alpha<\gamma \leqslant \beta$. Let $f$ be a real or complex valued functional defined on $C_{1}[a, b]$ such that $f(x(\cdot, \gamma))$ is a Yeh-Wiener measurable functional of $x(\cdot, \cdot)$ on $C_{2}[R]$. Then $f\left([(\gamma-\alpha) / 2]^{1 / 2} y\right)$ is a Wiener measurable functional of $y$ on $C_{1}[a, b]$ and

$$
\int_{C_{2}[R]} f(x(\cdot, \gamma)) d x=\int_{C_{1}[a, b]} f\left([(\gamma-\alpha) / 2]^{1 / 2} y\right) d y
$$

where the existence of either integral implies the existence of the other and their equality.

Proof. Case 1. Let $f(y)=\chi_{A}(y)$ where $A$ is a subset of $C_{1}[a, b]$ such that $\chi_{A}(x(\cdot, \gamma))$ is a Yeh-Wiener measurable functional of $x(\cdot, \cdot)$ on $C_{2}[R]$ (i.e. $f(y)=\chi_{A}(y)$ where $\left.B_{A} \in \Re_{2}\right)$.

Using Theorem 1 we immediately obtain that the functional

$$
\left.f([\gamma-\alpha) / 2]^{1 / 2} y\right)=\chi_{A}\left([(\gamma-\alpha) / 2]^{1 / 2} y\right)=\chi_{[2 /(\gamma-\alpha)]^{1 / 2} A}(y)
$$

is Wiener measurable and (4) follows from (3).

Case 2. Let $f(y)$ be a simple functional such that $f(x(\cdot, \gamma))$ is a Yeh-Wiener measurable functional of $x(\cdot, \cdot)$ on $C_{2}[R]$.

This case follows easily from Case 1 since we can write $f(y)$ in the form $f(y)=\sum_{j=1}^{n} c_{j} \chi_{A_{j}}(y)$ where each $c_{j}$ is real and each $\chi_{A_{j}}(y)$ is of the type considered in Case 1 .

Case 3. Let $f(y)$ be a real nonnegative functional such that $f(x(\cdot, \gamma))$ is a Yeh-Wiener measurable functional of $x(\cdot, \cdot)$ on $C_{2}[R]$.

In this case $f(y)$ is the limit of a monotone increasing sequence of simple functions and so the desired conclusions follow from Case 2 and the monotone convergence theorem.

Case 4. General case.

This case follows from Case 3 since we can decompose any complex functional into its real and imaginary parts and then into their positive and negative parts.

3. Proof of Theorem 1. The proof of Theorem 1 will follow quite readily once we establish three lemmas.

Definition. Let $\delta$ be a fixed constant satisfying $0<\delta<\frac{1}{2}$ and let $h>0$ be given. Let

$$
\begin{aligned}
A_{h} \equiv A_{h}(\delta) \equiv\{x & \in C_{2}[R]|| x\left(s_{2}, t_{2}\right)-x\left(s_{1}, t_{1}\right) \mid \\
& \leqslant h\left[\left(s_{2}-s_{1}\right)^{2}+\left(t_{2}-t_{1}\right)^{2}\right]^{\delta / 2} \\
& \left.\quad \text { for all } s_{1}, s_{2} \in[a, b] \text { and }\left(t_{1}, t_{2}\right) \in[\alpha, \beta]\right\} .
\end{aligned}
$$

LeMmA 1. (a) For any $\varepsilon>0$ there exists $h_{0}>0$ such that $m_{2}\left(A_{h}^{c}\right)<\varepsilon$ for all $h \geqslant h_{0}$. In fact $m_{2}\left(\cup_{h=1}^{\infty} A_{h}\right)=1$. (b) For each $h>0, A_{h}$ is compact in the uniform topology.

Proof. Statement (a) was established by Yeh [4, Theorem 1]. In [4, Lemma 5] Yeh showed that $A_{h}$ was compact in itself in the weak topology on $C_{2}[R]$ i.e. for any sequences $\left\{x_{n}\right\}$ in $A_{h}$ there exists a subsequence $\left\{x_{n_{k}}\right\}$ which 
converges pointwise on $R$ to an element $x_{0}$ in $A_{h}$. But $A_{h}$ is equicontinuous and equibounded. Hence by Ascoli's Theorem every sequence of elements from $A_{h}$ has a subsequence that converges uniformly. But $A_{h}$ is closed in the uniform topology and hence is sequentially compact in the uniform topology. But the uniform topology is a metric topology and so $A_{h}$ is compact in the uniform topology on $C_{2}[R]$.

Lemma 2. Let $\alpha<\gamma \leqslant \beta$, let $A$ be any subset of $C_{1}[a, b]$ and let $B_{A}$ $=\left\{x \in C_{2}[R] \mid x(\cdot, \gamma) \in A\right\}$. Let $G$ be any open set in $C_{2}[R]$ containing $B_{A}$. Let $h>0$ be given. Then there exists an open set $U$ in $C_{1}[a, b]$ such that $A \subseteq U$ and $\left(A_{h} \cap B_{U}\right) \subseteq G$ where $B_{U}=\left\{x \in C_{2}[R] \mid x(\cdot, \gamma) \in U\right\}$.

Proof. Case 1. Assume $A$ consists of just one point, say $y_{0}(\cdot)$. Assume Lemma 2 is false. Then there exists a sequence of points $\left\{x_{n}(\cdot, \cdot)\right\}_{n=1}^{\infty}$ in $A_{h}-G$ such that

$$
\lim _{n \rightarrow \infty} x_{n}(s, \gamma)=y_{0}(s) \text { for each } s \in[a, b] .
$$

Since $A_{h}$ is compact in the uniform topology there exists a subsequence $\left\{x_{n_{k}}(\cdot, \cdot)\right\}_{k=1}^{\infty}$ which converges uniformly, say to $x_{0}(\cdot, \cdot)$, on $R$. Thus $\lim _{k \rightarrow \infty} x_{n_{k}}(s, \gamma)=x_{0}(s, \gamma)$ for all $s \in[a, b]$ and so $x_{0}(\cdot, \gamma)=y_{0}(\cdot)$. Hence $x_{0} \in B_{A}$. But $G^{c}$ is a closed set in $C_{2}[R]$ and so $x_{0} \in G^{c} \subseteq B_{A}^{c}$ which is contrary to $x_{0} \in B_{A}$. Hence Lemma 2 is established when $A$ is a singleton set.

Case 2. General case. Let $A$ be any set in $C_{1}[a, b]$. Using Case 1 we see that for each point $y$ in $A$ there exists an open set $U_{y}$ in $C_{1}[a, b]$ containing $y$ such that $\left(A_{h} \cap B_{U_{y}}\right) \subseteq G$. Then $U \equiv \cup_{y \in A} U_{y}$ is an open set in $C_{1}[a, b]$ containing $A$ such that $\left(A_{h} \cap B_{U}\right) \subseteq G$.

Lemma 3. Let $\gamma, A$ and $B_{A}$ be as in Lemma 2. Then

$$
m_{y}^{*}\left(B_{A}\right)=m_{1}^{*}\left([2 /(\gamma-\alpha)]^{1 / 2} A\right) .
$$

Proof. (i) We will first show that $m_{2}^{*}\left(B_{A}\right) \leqslant m_{1}^{*}\left([2 /(\gamma-\alpha)]^{1 / 2} A\right)$. Let $\hat{A}$ be a subset of $C_{1}[a, b]$ such that $A \subseteq \hat{A},[2 /(\gamma-\alpha)]^{1 / 2} \hat{A} \in \mathfrak{R}_{1}$ and

$$
m_{1}\left([2 /(\gamma-\alpha)]^{1 / 2} \hat{A}\right)=m_{1}^{*}\left([2 /(\gamma-\alpha)]^{1 / 2} A\right) .
$$

Then $B_{\hat{A}} \in \Re_{2}, B_{A} \subseteq B_{\hat{A}}$ and

$$
\begin{aligned}
m_{2}^{*}\left(B_{A}\right) & \leqslant m_{2}^{*}\left(B_{\hat{A}}\right)=m_{2}\left(B_{\hat{A}}\right)=m_{1}\left([2 /(\gamma-\alpha)]^{1 / 2} \hat{A}\right) \\
& =m_{1}^{*}\left([2 /(\gamma-\alpha)]^{1 / 2} A\right) .
\end{aligned}
$$

(ii) Let $\varepsilon>0$ be given. We need only show that $m_{1}^{*}\left([2 /(\gamma-\alpha)]^{1 / 2} A\right)$ $\leqslant m_{2}^{*}\left(B_{A}\right)+\varepsilon$. First choose $H \in \mathfrak{N}_{2}$ such that $B_{A} \subseteq H$ and $m_{2}^{*}\left(B_{A}\right)$ $=m_{2}(H)$. Next choose $h>0$ so large that $m_{2}\left(A_{h}^{c}\right)<\varepsilon / 2$. Then

$$
m_{2}\left(H \cup A_{h}^{c}\right) \leqslant m_{2}(H)+m_{2}\left(A_{h}^{c}\right)<m_{2}^{*}\left(B_{A}\right)+\varepsilon / 2 .
$$

Now [4, Lemma 6] let $G$ be an open subset of $C_{2}[R]$ such that $\left(H \cup A_{h}^{c}\right) \subseteq G$ and $m_{2}\left(G-\left(H \cup A_{h}^{c}\right)\right)<\varepsilon / 2$. Then 


$$
m_{2}(G)<m_{2}\left(H \cup A_{k}^{c}\right)+\varepsilon / 2 .
$$

Next, by Lemma 2 above, there exists an open set $U$ in $C_{1}[a, b]$ such that $A \subseteq U$ and $\left(A_{h} \cap B_{U}\right) \subseteq G$. But

$$
\left(B_{U} \cap A_{h}^{c}\right) \subseteq A_{h}^{c} \subseteq\left(H \cup A_{h}^{c}\right) \subseteq G
$$

and so $B_{U}=\left(B_{U} \cap A_{h}^{c}\right) \cup\left(B_{U} \cap A_{h}\right) \subseteq G$. Now since $U$ is an open set in $C_{1}[a, b]$ it follows that $[2 /(\gamma-\alpha)]^{1 / 2} U \in \mathfrak{R}_{1}$. But this implies that $B_{U} \in \mathfrak{R}_{2}$ and the equality $m_{1}\left([2 /(\gamma-\alpha)]^{1 / 2} U\right)=m_{2}\left(B_{U}\right)$. Hence using (8), (7), and (6) we obtain

$$
\begin{aligned}
m_{1}^{*}\left([2 /(\gamma-\alpha)]^{1 / 2} A\right) & \leqslant m_{1}\left([2 /(\gamma-\alpha)]^{1 / 2} U\right) \\
& =m_{2}\left(B_{U}\right) \leqslant m_{2}(G)<m_{2}^{*}\left(B_{A}\right)+\varepsilon
\end{aligned}
$$

which establishes (5).

Proof (OF Theorem 1). We only need to show that $B_{A} \in \pi_{2}$ implies $[2 /(\gamma-\alpha)]^{1 / 2} A \in \mathfrak{R}_{1}$. So assume that $B_{A} \in \mathfrak{N}_{2}$. Then by Lemma 3

$$
m_{1}^{*}\left([2 /(\gamma-\alpha)]^{1 / 2} A\right)=m_{2}^{*}\left(B_{A}\right)=m_{2}\left(B_{A}\right) .
$$

Now $B_{A} \in \Re_{2}$ implies that $\left(B_{A}\right)^{c} \in \Re_{2}$ and since

$$
\left(B_{A}\right)^{c}=B_{A^{c}} \text { and }\left([2 /(\gamma-\alpha)]^{1 / 2} A\right)^{c}=[2 /(\gamma-\alpha)]^{1 / 2} A^{c},
$$

another application of Lemma 3 yields

$$
\begin{aligned}
m_{1}^{*}\left(\left([2 /(\gamma-\alpha)]^{1 / 2} A\right)^{c}\right) & =m_{1}^{*}\left([2 /(\gamma-\alpha)]^{1 / 2} A^{c}\right) \\
& =m_{2}^{*}\left(B_{A^{c}}\right)=m_{2}^{*}\left(\left(B_{A}\right)^{c}\right)=m_{2}\left(\left(B_{A}\right)^{c}\right) .
\end{aligned}
$$

Hence using (9) and (10) we obtain that

$$
m_{1}^{*}\left([2 /(\gamma-\alpha)]^{1 / 2} A\right)+m_{1}^{*}\left(\left([2 /(\gamma-\alpha)]^{1 / 2} A\right)^{c}\right)=1
$$

from which it follows that $[2 /(\gamma-\alpha)]^{1 / 2} A \in \mathfrak{N}_{1}$.

4. Koehler's Theorem for $C_{2}[R]$.

THEOREM 4. Let $a=s_{0}<s_{1}<\cdots<s_{m}=b$ and $\alpha=t_{0}<t_{1}<\cdots$ $<t_{n}=\beta$ be subdivisions of $[a, b]$ and $[\alpha, \beta]$ respectively. Let $E$ be any subset of Euclidean space $R^{m n}$ and let

$$
Q=\left\{x \in C_{2}[R] \mid\left\langle x\left(s_{1}, t_{1}\right), \ldots, x\left(s_{m}, t_{n}\right)\right\rangle \in E\right\} .
$$

If $Q \in \mathfrak{N}_{2}$ then $E$ is a Lebesgue measurable subset of $R^{m n}$.

Corollary. Let $f: R^{m n} \rightarrow C$ and let $F: C_{2}[R] \rightarrow C$ be defined by $F(x)$ $=f\left(x\left(s_{1}, t_{1}\right), \ldots, x\left(s_{m}, t_{n}\right)\right)$. If $F(x)$ is a Yeh-Wiener measurable functional on $C_{2}[R]$ then $f\left(u_{1,1}, \ldots, u_{m, n}\right)$ is Lebesgue measurable on $R^{m n}$.

The proof of Theorem 4 follows quite easily once Lemma 5 below is established. We will omit the proofs of Lemmas 4 and 5 below since they are similar to the proofs given above for Lemmas 2 and 3 respectively. 
Lemma 4. Let $E$ and $Q$ be as in Theorem 4. Let $G$ be any open set in $C_{2}[R]$ containing $Q$. Let $h>0$ be given. Then there exists an open set $U$ in $R^{m n}$ containing $E$ such that

$$
\left\{x \in C_{2}[R] \mid x \in A_{h} \text { and }\left\langle x\left(s_{1}, t_{1}\right), \ldots, x\left(s_{m}, t_{n}\right)\right\rangle \in U\right\} \subseteq G .
$$

Definition. We define a probability measure $v$ on the Lebesgue measurable subsets $E$ of $R^{m n}$ by

$$
v(E) \equiv \int_{E} W(\vec{u} ; \vec{s} ; \vec{t}) d \vec{u}
$$

where $W(\vec{u} ; \vec{s} ; \vec{t})$ is given by (2). Let $v^{*}$ be the usual regular outer measure based on $v$ such that $v^{*}$ is defined for all subsets of $R^{m n}$.

Lemma 5. Let $E$ be any subset of $R^{m n}$ and let $Q$ be defined by (11). Then $m_{2}^{*}(Q)=v^{*}(E)$.

\section{BIBLIOGRAPHY}

1. R. H. Cameron and W. T. Martin, The behavior of measure and measurability under change of scale in Wiener space, Bull. Amer. Math. Soc. 53 (1947), 130-137. MR 8, 392.

2. R. H. Cameron and D. A. Storvick, Two related integrals over spaces of continuous functions, Pacific J. Math. 55 (1974), 19-38.

3. - An operator valued Yeh-Wiener integral, and a Wiener integral equation, Indiana Univ. Math. J. 25 (1976), 235-258.

4. J. Yeh, Wiener measure in a space of functions of two variables, Trans. Amer. Math. Soc. 95 (1960), 433-450. MR 23 \# A2735.

Department of Mathematics and Statistics, University of Nebraska, Lincoln, Nebraska 68588 